\title{
Common mode chokes and optimisation aspects
}

\author{
T. Kut ${ }^{1}$, A. Lücken ${ }^{2}$, S. Dickmann ${ }^{1}$, and D. Schulz ${ }^{2}$ \\ ${ }^{1}$ Institute of Fundamentals of Electrical Engineering, Helmut Schmidt University, Hamburg, Germany \\ ${ }^{2}$ Institute of Electrical Power Systems, Helmut Schmidt University, Hamburg, Germany
}

Correspondence to: T. Kut (tobias.kut@hsu-hh.de)

Received: 3 January 2014 - Accepted: 29 January 2014 - Published: 10 November 2014

\begin{abstract}
Due to the increasing electrification of modern aircraft, as a result of the More Electric Aircraft concept, new strategies and approaches are required to fulfil the strict EMC aircraft standards (DO-160/ED-14-Sec. 20). Common mode chokes are a key component of electromagnetic filters and often oversized because of the unknown impedance of the surrounding power electronic system. This oversizing results in an increase of weight and volume. It has to be avoided as far as possible for mobile applications. In this context, an advanced method is presented to measure these impedances under operating conditions. Furthermore, the different parameters of the inductance design is explained and an optimisation for weight and volume is introduced.
\end{abstract}

\section{Introduction}

Power electronic converters, for example an interleaved boost converter as shown in Fig. 1, consist of switching devices, which are turned on or off, based on a modulation scheme to adjust the output voltage. The main drawback is the generated level of electromagnetic interferences on power lines and other communication wires due to the non-ideal behaviour of the used semiconductors (Farhadi and Jalilian, 2006; Mainali and Oruganti, 2007). The spectrum is usually spread from $10 \mathrm{kHz}$ to $100 \mathrm{MHz}$. Filtering the transmission wires is a challenge, which often leads to redesigned filters, tested in a "trial and error" procedure and optimised only rarely in terms of weight, size or costs (Mayer, 1998). These optimisations are required, especially for applications in modern aircraft (Derouineua, 2009; Kut et al., 2013a; Chand et al., 2013). Therefore, an advanced method is presented to measure the impedances of the power converter to improve the performance of the filter (Tarateeraseth et al., 2011; See and Deng, 2004; Kut et al., 2013b). Fur-

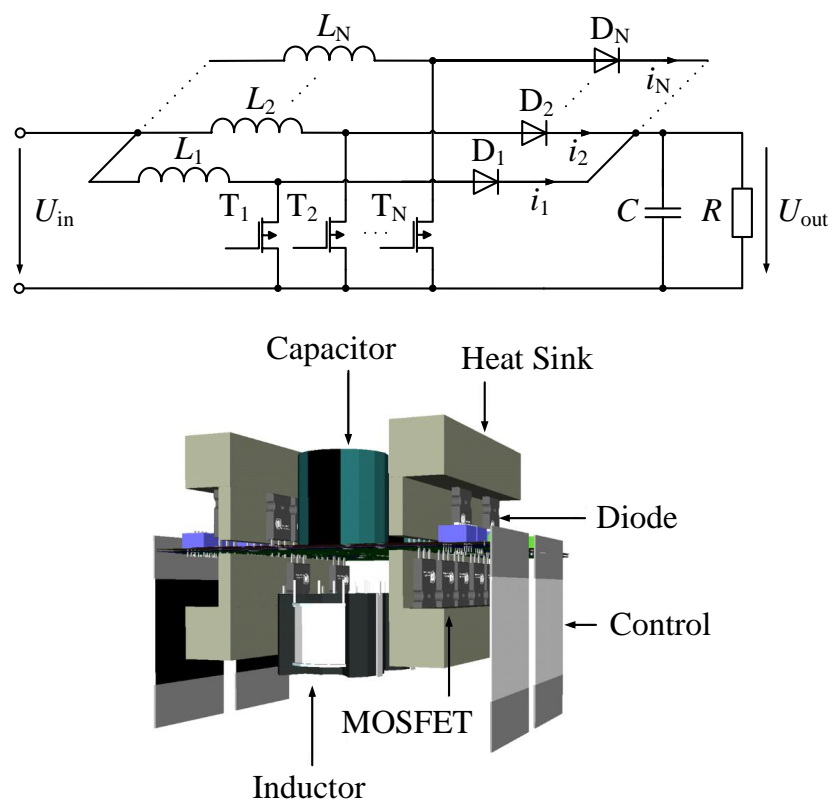

Figure 1. Schematic of a boost converter in interleaved structure (above) with prototypical realization (3-D-model) as four-step interleaved converter (below) as an exemplary application for modern aircraft.

thermore, the different design parameters such as frequency, saturation flux density and operating current are compared for various new ferromagnetic materials (Vacuumschmelze ${ }^{\circledR}$, 2013; Magnetics ${ }^{\circledR}, 2013$ ). These approaches may reduce or avoid the need for construction of several, often oversized, filter prototypes. They provide an additional insight into the behaviour of the choke by giving an understanding, how variations of parameters influence the final filter performance. 


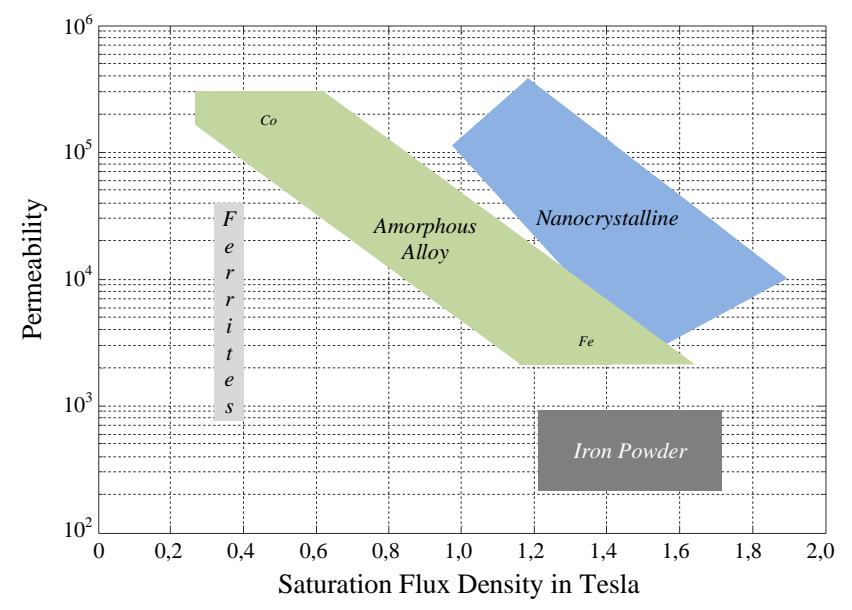

Figure 2. Magnetic properties for ferrites, iron powder and metal alloys: permeability vs. saturation flux density (Roc'h and Leferink, 2013).

\section{Designable parameters and impedance modelling}

This section focuses on the designable parameters of a common mode choke or a storage inductor such as core material, operating frequency, saturation and impedance behaviour.

\subsection{Core material}

The main purpose of common mode chokes is to transform the electromagnetic interferences to heat rather than for energy storage as a storage inductor. The application of the core material is quite similar. The material can be used to increase the inductance, to absorb energy and to transform it to heat. The permeability $\mu$ as the ratio of the magnetic flux density $B$ to $H$ is the most important magnetic material parameter. Typical requirements of an optimised choke are a high saturation level and a wide frequency range. Figure 2 shows the dependence of the permeability as a function of the saturation flux density and Fig. 3 of the frequency, respectively. Three basic materials, i.e. ferrites, iron powder and metal alloys (nanocrystalline and amorphous structure) are used in the design of traditional common mode chokes. The highest permeabilities are found in nanocrystalline materials. Amorphous alloys have somewhat smaller values whereas iron powder cores have relatively low permeabilities. Ferrite cores can be used over a wide frequency range but exhibit a significantly lower level of saturation. Higher flux densities can be found in nanocrystalline and amorphous materials as well as in iron powder cores but are limited in the used frequency range. Nanocrystalline materials are a good alternative to the traditional chokes made of ferrite or iron powder. So, the main advantage is the high level of saturation and the resulting smaller size. The use of this material as a filter element or the use as a storage inductor due to the distributed air gap, in contrast to a conventional ferrite core is a step towards vol-

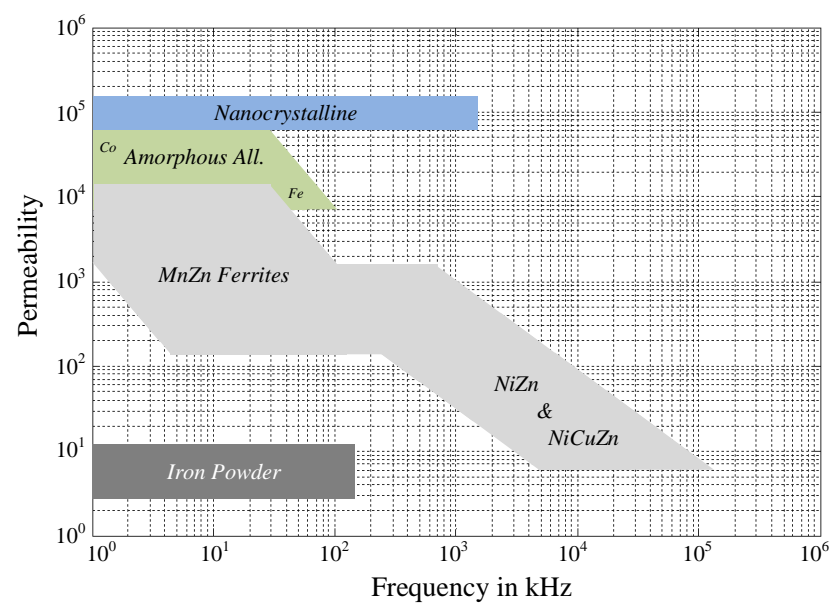

Figure 3. Magnetic properties for ferrites, iron powder and metal alloys: permeability vs. frequency (Roc'h and Leferink, 2013).

ume reduction. Reaching 50-80\% lower compared to a ferrite volume and more than $80 \%$ lower compared to an iron core. The resulting weight reduction is an important property in aircraft applications or any other areas where weight is a design constraint (Wen, 2011).

\subsection{Impedance modelling}

A main objective of a common mode choke is to block the electromagnetic interferences. The study of the behaviour involves five types of impedances. Figure 4 presents the general equivalent circuit with the coupling factor $K$ :

- the common mode $\left(L_{\mathrm{cm}}\right)$

- the differential mode $\left(L_{\mathrm{dm}}\right)$

- the inter-winding $\left(C_{\mathrm{p}}\right)$

- the intra-winding $\left(C_{\mathrm{w}}\right)$

- the line impedance $\left(R_{\text {wire }}\right)$

Equation (1), the value of the common mode impedance is the most important value and is strongly related to the permeability (assuming linearity) of the core. In addition to the operating current, the inductance and the capacitance - the designable parameters in these context are the core material $\left(\mu=\mu_{0}\left(\mu_{\mathrm{RL}}^{\prime}-\mathrm{j} \mu_{\mathrm{RR}}^{\prime \prime}\right)\right)$, the dimension and configuration ( $A_{\text {mat }}$ ), the wire material (frequency, diameter, isolation), the number of turns $(N)$ and the effective length $\left(l_{\text {mat }}\right)$. This can be modified by the designer and the impedance can be represented by a series equivalent circuit consisting of an ideal inductor $L_{\text {ser }}$ and a resistor $R_{\text {ser }}$. 


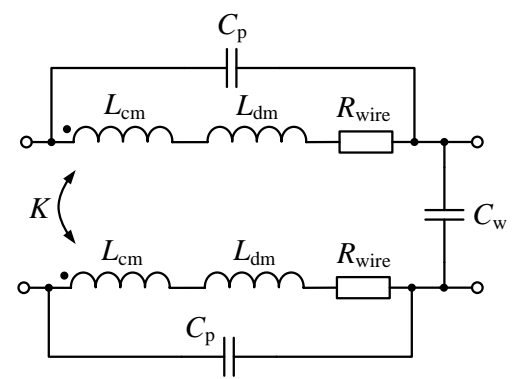

Figure 4. Equivalent circuit of the common mode choke.

$$
\begin{gathered}
\underline{Z}_{\mathrm{cm}}=\mathrm{j} \omega L_{\mathrm{cm}}= \\
=\mathrm{j} \omega \underbrace{\frac{\mu_{0} \cdot \mu_{\mathrm{RL}}^{\prime} \cdot A_{\mathrm{mat}} \cdot N^{2}}{l_{\mathrm{mat}}}}_{L_{\mathrm{ser}}}+\underbrace{\omega \frac{\mu_{0} \cdot \mu_{\mathrm{RR}}^{\prime \prime} \cdot A_{\mathrm{mat}} \cdot N^{2}}{l_{\text {mat }}}}_{R_{\mathrm{ser}}} \\
L_{\mathrm{cm}}=\mu_{0}\left(\mu_{\mathrm{RL}}^{\prime}-\mathrm{j} \mu_{\mathrm{RR}}^{\prime \prime}\right) \cdot \frac{A_{\mathrm{mat}} \cdot N^{2}}{l_{\mathrm{mat}}}
\end{gathered}
$$

\subsection{Measurements}

In order to evaluate the influence of each parameter, measurements with a precision LCR meter with dc bias unit are performed for various core materials, which can be assigned to the respective classes of materials. The influence of the frequency (Fig. 5 for $f=100 \mathrm{kHz}$, Fig. 6 for $f=300 \mathrm{kHz}$ ) and the flux density is presented. Only the suitable materials have been measured for the higher frequency in Fig. 6 . The maximum saturation flux density $B_{\max }$ ranges from $0.5 \mathrm{~T}$ (PM 50/39) to 2T (HFlux) and can be described by Eq. (2) with the maximum operating current $\hat{I}_{\max }$, the minimum $A_{\min }$ and effective cross-sectional area $A_{\text {eff }}$ for ferrite cores and with the material-dependent inductance factor $A_{\mathrm{L}}$ for toroidal cores.

$B_{\max }=\underbrace{\frac{N \cdot \hat{I}_{\max }}{\frac{l_{\text {mat }}}{\mu_{0} \cdot \mu_{\text {mat }}}+\frac{l_{\text {air }}}{\mu_{0}}} \cdot \frac{A_{\text {eff }}}{A_{\text {min }}}}_{\text {Ferrites }}=\underbrace{\frac{N \cdot \hat{I}_{\text {max }} \cdot A_{\mathrm{L}}}{A_{\text {mat }}}}_{\text {Toroid }}$

As already mentioned, the common mode chokes, inductors or storage inductors constitute a high influence in power electronic systems. The design of these elements has been a challenging task with the increasing complexity of the power electronic. In order to optimize this in the context of weight savings, the area product method is used. This is defined as the product of the core area $A_{\text {core }}$ and the window area $A_{\text {window }}$ for a core - according to Eq. (3). The various parameters involved in the design are divided into three categories: design requirements, material and manufacturing constraints. Here, the value $A P_{\min }$ can be determined and should be minimised from the output power $P$ and operating frequency

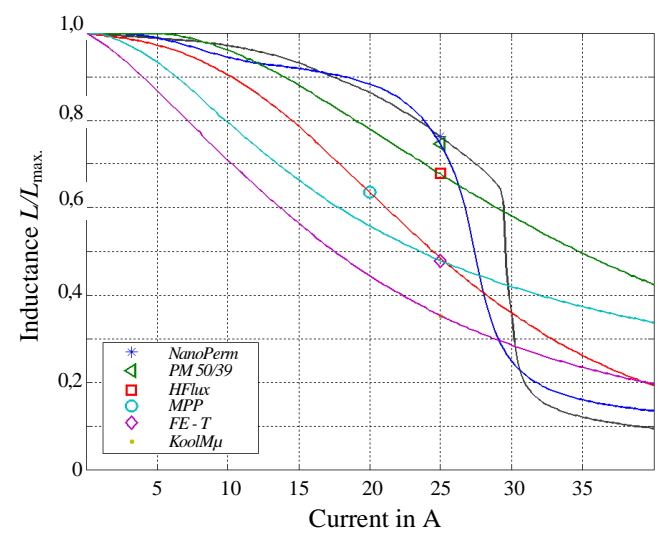

Figure 5. Core materials at $f=100 \mathrm{kHz}$.

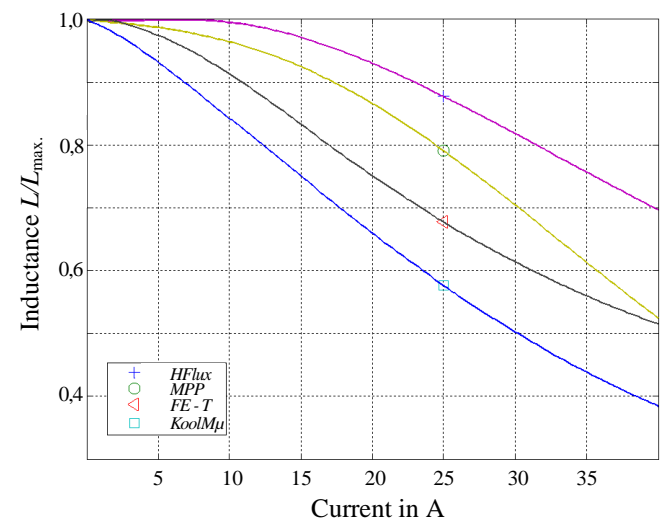

Figure 6. Core materials at $f=300 \mathrm{kHz}$.

$f$ as design requirements, the maximum flux density $B_{\max }$ and maximum current density $J_{\max }$ as material constraints and the window utilisation factor $C$ as manufacturing constraint. The area product method is a very useful expression involving mechanical and electrical parameters. The increase of the operating frequency and the material-dependent saturation flux density contribute to the weight saving effect.

$A P_{\min }=A_{\text {core }} \cdot A_{\text {window }}=\frac{2 \cdot P}{C \cdot B_{\max } \cdot f \cdot J_{\max }}$

In addition to the relationship of the inductance as a function of the flux density and the frequency, the impedance of the common mode choke is another important parameter for the attenuation of electromagnetic interferences. For the common mode impedance measurements the two input and the two output ports with respect to Fig. 4 are connected to each other. Therefore, the impedances have been measured for various cores (Fig. 8) with an impedance analyser between $9 \mathrm{kHz}$ and $100 \mathrm{MHz}$. The result is presented in Fig. 7. For comparison, two conventional filter elements $\left(\right.$ VitroVac ${ }^{\circledR}$, NanoPerm $^{\circledR}$ ) are compared with the new materials. It is clear that, for example, AmoFlux can also be used as a filter element, which has a significantly lower weight as 


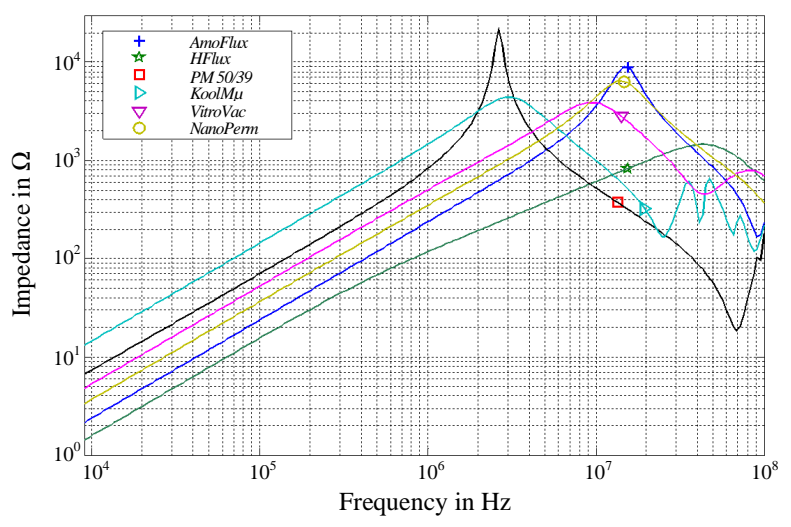

Figure 7. Measured common mode impedance for various cores.

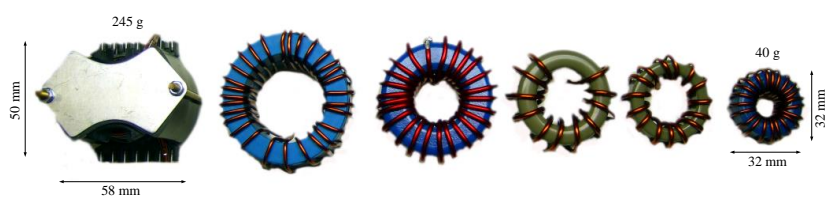

Figure 8. Overview about the core materials.

indicated in Fig. 8. In summary, it can be noted that weight can be saved by the choice of newer materials for common mode chokes as well as for storage inductors. A further advantage is that they have a higher saturation flux density and can also be used for a much higher frequency range.

\section{System impedance measurement}

An accurate measurement method to extract the common and differential mode impedance of a power electronic system under operating conditions is presented in this section. For this purpose a pre-measurement calibration process and the extraction of common and differential mode is described to systematically design an electromagnetic filter with minimum effort. The system impedance is very important for filter design because only with this information it can be best adapted to the system, without oversizing the filter. In contrast to other systems due to converter topology, component parasitics, printed circuit board layout etc. the impedance is not $50 \Omega$ and must be taken into account. For example, the differential mode is influenced by the reverse recovery effect of the diode or the equivalent series resistance and inductance of a capacitor. As for the common mode, the deciding components are parasitic effects between the semiconductor, heat-sink, chassis and printed circuit board. To illustrate, why the system impedances are important parameters for filter design, the simple schematic as shown in Fig. 9 is analysed by Eq. (4), which represents the transfer function of the entire system. The parameters $a_{\mathrm{xy}}$ are the elements of a cascaded filter structure. This clearly illustrates that for optimum EMI filter design, the impedances of source and load should be

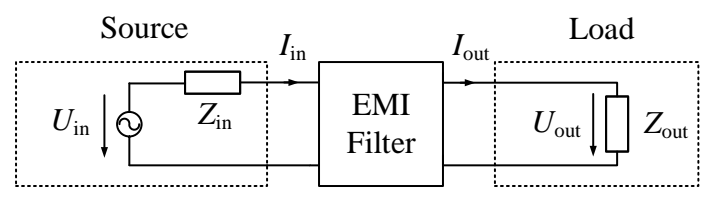

Figure 9. Consideration of the system impedances in filter design.

taken into account in power electronic applications. A detailed description is given by (Kut et al., 2013b).

$\frac{U_{\text {out }}}{U_{\text {in }}}=\frac{1}{a_{11}+\frac{a_{12}}{Z_{\text {out }}}+a_{21} \cdot Z_{\text {in }}+a_{22} \cdot \frac{Z_{\text {in }}}{Z_{\text {out }}}}$

Therefore, a two-probe measurement procedure for common and differential mode impedances is advantageous for power electronic systems. It was originally developed for built-in power line electromagnetic filters as small-signal behaviour (Tarateeraseth et al., 2011). It has been extended as follows:

- Measurements under operating conditions in each operating point of the power electronic system

- Independent of each used measurement tool (calibrated out of cables, current probes etc.)

- Minimal frequency range from $9 \mathrm{kHz}$ to $30 \mathrm{MHz}$

- Separation between common and differential mode

- Operating parameters of several hundred volts and several tens of amperes

The basic concept of the method to measure the unknown impedance $Z_{X}$ is shown in Fig. 10 (above). It consists of an injection probe, a detection current probe, a vector network analyser (VNA) and the unknown impedance. Port 1 generates a signal into closed-loop through the injection probe and the resulting current is measured at port 2. Figure 10 (below) shows the complete equivalent circuit of the measurement setup with port 1 as source $U_{1}$ as well as the internal resistance $Z_{\mathrm{i}}$, the source voltage $U_{\mathrm{C} 1}$ as well as the resulting voltage $U_{\mathrm{C} 2}$, the primary $L_{1} / L_{2}$ and the mutual inductances $M_{1} / M_{2}$, the formed wiring elements $L_{\mathrm{w}}$ and $R_{\mathrm{w}}$. These variables are summarised in addition to $Z_{\text {setup }}$ (Kut et al., 2013b). By solving the Eq. (5) as equation of the system as indicated in Fig. 10 (below), the unknown impedance $Z_{X}$ can be expressed by Eq. (6), where $F$ is a frequency-independent factor $\left(Z_{\mathrm{T}}\right.$ is the ratio of $U_{\mathrm{C} 2}$ and $\left.I_{\text {out }}\right)$. The ratio of the twoprobe voltages is given by Eq. (7).

$$
\begin{gathered}
\left(\begin{array}{c}
U_{1} \\
0 \\
-Z_{\mathrm{X}} \cdot I_{\text {out }}
\end{array}\right)= \\
=\left(\begin{array}{ccc}
Z_{\mathrm{i}}+\mathrm{j} \omega L_{1} & 0 & -\mathrm{j} \omega M_{1} \\
0 & Z_{\mathrm{i}}+\mathrm{j} \omega L_{2} & j \omega M_{2} \\
-\mathrm{j} \omega M_{1} & j \omega M_{2} & R_{\mathrm{w}}+\mathrm{j} \omega L_{\mathrm{w}}
\end{array}\right) \cdot\left(\begin{array}{c}
I_{1} \\
I_{2} \\
I_{\text {out }}
\end{array}\right)
\end{gathered}
$$




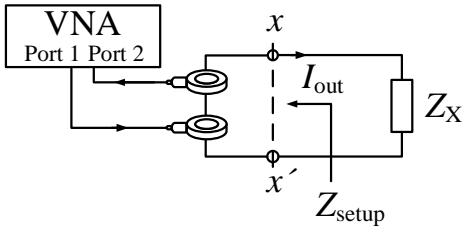

Equivalent circuit

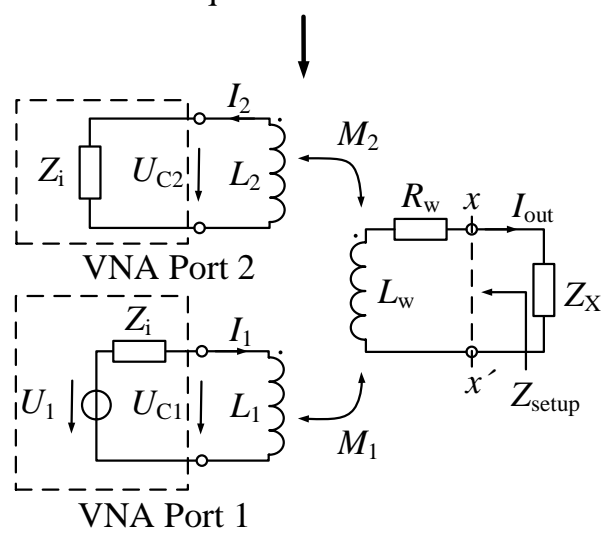

Figure 10. Basic concept and equivalent circuit of the measurement setup.

$Z_{\mathrm{X}}=F \cdot\left(\frac{U_{\mathrm{C} 1}}{U_{\mathrm{C} 2}}\right)-Z_{\text {setup }} \quad ; \quad F=\frac{M_{1} \cdot Z_{\mathrm{T}}}{L_{1}}$

$\frac{U_{\mathrm{C} 1}}{U_{\mathrm{C} 2}}=\frac{S_{11}+1}{S_{21}}$

The coefficient $F$ and the setup impedance $Z_{\text {setup }}$ can be obtained by two additional measurements: First, by replacing the unknown impedance $Z_{X}$ in Fig. 10 with a known precision resistor $R$. The resistor should be chosen in the middle range of the unknown impedance to be measured. The next measurement should be done without the resistor $R$. Therefore, $R$ can be short-circuited in Fig. 10. With these additional measurements and Eqs. (5) and (6), $F$ and the setup impedance $Z_{\text {setup }}$ can be obtained by solving this system of equations. All parameters, which are influenced by the measurement setup are determined and can be eliminated for the determination of $Z_{X}$. The measurement setup to extract the "unknown" common mode impedance is shown in Fig. 11. In order to avoid any unwanted electromagnetic interferences from the source supplying the power electronic, these connections are terminated by line impedance stabilisation networks (LISN). Basically, the setup and the connecting wires should correspond to those of the calibration measurements. It is the same procedure with the electronic load to be supplied in Fig. 11. To measure the differential mode impedance one of the two wires must be rotated by $180^{\circ}$ and routed back through the two current probes. Figure 12 shows exemplary the magnitude of the extracted common mode impedance. The intermediate steps for determining the $Z_{\text {setup }}$ and differential mode impedance are omitted for clarity, at this point.

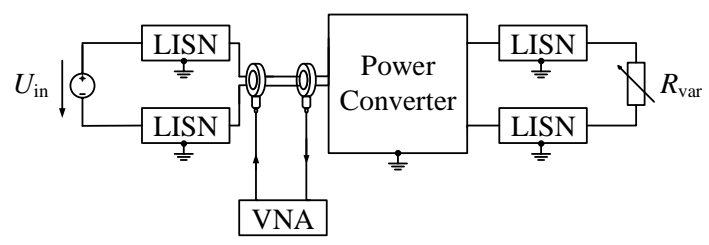

Figure 11. Setup for common mode impedance measurement.

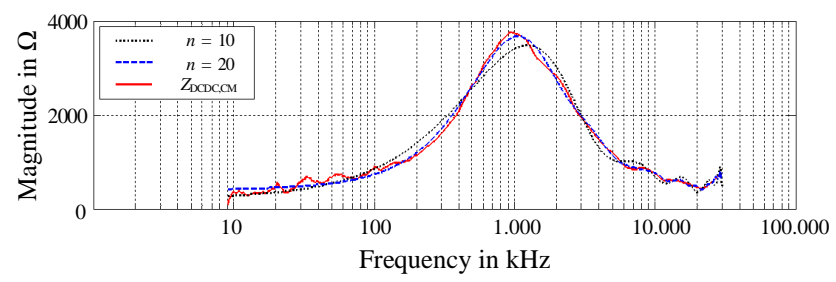

Figure 12. Measured common mode impedance with interpolated values of polynomial $n=10$ and $n=20$.

It can also be seen in Fig. 12 that for calculation of the filter elements, the measured curve is represented by a polynomial function, in order to use them in Eq. (4). In summary, with accurate measurements of common and differential mode impedances over the frequency range of interest, an appropriate filter can be chosen or can be designed with optimal component values and is no longer overdimensioned.

\section{Conclusions}

The EMC requirements increased as a result of the required DC/DC converters due to the installation of electric system components instead of heavy mechanical, pneumatic and hydraulic systems in modern aircraft. In order that these filters do not take up a large part of the overall system weight, they must be optimally adjusted to the converter system. Two aspects should be considered: on the one hand there are weight savings possible by the optimised selection of the inductor material. For this purpose, the dependencies with respect to frequency and saturation flux density for various modern materials have been identified and the potential savings were clearly pointed out. In addition, an accurate measurement method to extract common mode and differential mode impedance of power electronic converters under operation conditions was presented. With these measurements and with the knowledge of the correct material an appropriate EMI filter can be chosen or can be designed with optimal component values and is no longer overdimensioned.

Edited by: F. Sabath

Reviewed by: T. Ehlen and H. Garbe 


\section{References}

Chand, B., Kut, T., and Dickmann, S.: Optimal design of active EMC filters, Adv. Radio Sci., 11, 243-249, doi:10.5194/ars-11243-2013, 2013.

Derouineua, J.-L.: Power Optimized More Electrical Aircraft, in: Proceedings of the European Conference towards eEnvironment, 293-295, Prag, 2009.

Farhadi, A. and Jalilian, A.: Modeling and Simulation of Electromagnetic Conducted Emission Due to Power Electronics Converters, in: Power Electronics, Drives and Energy Systems, International Conference on, 1-6, New Delhi, 2006.

Kut, T., Chand, B., Lücken, A., Dickmann, S., and Schulz, D.: EMC studies on systems with hybrid filter circuits for modern aircraft applications, Adv. Radio Sci., 11, 237-242, doi:10.5194/ars-11237-2013, 2013a.

Kut, T., Lücken, A., Dickmann, S., and Schulz, D.: Design von Gleich- und Gegentaktfiltern unter Berücksichtigung der Ein- und Ausgangsimpedanzen für Gleichspannungswandler im HVDC-Netz, in: 62. Deutscher Luft- und Raumfahrtkongress, urn:nbn:de:101:1-2013112910658, Stuttgart, September, 2013b.

Magnetics: A Critical Comparison of Ferrites with other Magnetic Materials, in: Technical Information, 2010.
Mainali, K. and Oruganti, R.: Simple Analytical Models to Predict Conducted EMI Noise in a Power Electronic Converter, in: Industrial Electronics Society, 33rd Annual Conference of the IEEE, 1930-1936, Taipei, 2007.

Mayer, F.: Electrical Power and Signal Distribution in Modern Aircrafts, combines Weight Advantages and EMC Compatibility, in: Electromagnetic Compatibility, IEEE International Symposium on, 1, 281-283, Denver, 1998.

Roc'h, A. and Leferink, F. B. J.: Analysis of Common Mode Inductors and Optimization Aspects, in: Power Electronics and Power Systems, Electromagnetic Interference Issues, Bentham Science Publishers, Australien, 3-37, ISBN 978-1-60805-240-0, 2012.

See, K. Y. and Deng, J.: Measurement of Noise Source Impedance of SMPS Using a Two Probes Approach, in: Power Electronics, IEEE Transactions on, 19, 862-868, Mai, 2004.

Tarateeraseth, V., See, K. Y., Wang, L. B., and Canavero, F. G.: Systematic Power Line EMI Filter Design for SMPS, in: EMC Europe, 586-591, York, September, 2011.

Vacuumschmelze GmbH \& Co. KG: Nanocrystalline Material in Common-Mode Chokes, in: Technical Information, 2013.

Wen, B.: Weight Estimation of Electronic Power Conversion Systems, in: Master's Thesis, Virginia Polytechnic Institute and State University, 2011. 